\title{
Development of a noninvasive system for monitoring dairy cattle sleep
}

\author{
J. M. Klefot, ${ }^{*}$ J. L. Murphy, ${ }^{*}$ K. D. Donohue,† B. F. O’Hara,‡ M. E. Lhamon,§ and J. M. Bewley ${ }^{\star 1}$ \\ *Department of Animal and Food Sciences, \\ †Center for Visualization and Virtual Environments, and \\ ‡Department of Biology, University of Kentucky, Lexington 40506 \\ $\S$ Digital Key Consulting, Lexington, KY 40515
}

\section{ABSTRACT}

Limited research has been conducted to assess sleep in production livestock primarily because of limitations with monitoring capabilities. Consequently, biological understanding of production circumstances and facility options that affect sleep is limited. The objective of this study was to assess if data collected from a proofof-concept, noninvasive 3 -axis accelerometer device are correlated with sleep and wake-like behaviors in dairy cattle. Four Holstein dairy cows housed at the University of Kentucky Coldstream Dairy in September 2013 were visually observed for 2 consecutive 24 -h periods. The accelerometer device was attached to a harness positioned on the right side of each cow's neck. Times of classified behaviors of wake (standing, head up, alert, eyes open) or sleep-like behaviors (lying, still, head resting on ground, eyes closed) were recorded continuously by 2 observers who each watched 2 cows at a time. The radial signal was extracted from 3 different axes of the accelerometer to obtain a motion signal independent of direction of movement. Radial signal features were examined for maximizing the performance of detecting sleep-like behaviors using a Fisher's linear discriminant analysis classifier. The study included $652 \mathrm{~min}$ of high-activity wake behaviors and 107 min of sleep-like behavior among 4 cows. Results from a bootstrapping analysis showed an agreement between human observation and the linear discriminant analysis classifier, with an accuracy of $93.7 \pm 0.7 \%$ for wake behavior and $92.2 \pm 0.8 \%$ for sleep-like behavior $( \pm 95 \%$ confidence interval). This prototype shows promise in measuring sleep-like behaviors. Improvements to both hardware and software should allow more accurate determinations of subtle head movements and respiratory movements that will further improve the assessment of these sleep-like behaviors, including estimates of deep, light, and rapid eye movement sleep. These future studies

Received November 29, 2015.

Accepted May 17, 2016.

${ }^{1}$ Corresponding author: jbewley@uky.edu will require simultaneous electroencephalography and electromyography measures and perhaps additional measures of arousal thresholds to validate this system for measuring true sleep.

Key words: behavior, sleep, accelerometer

\section{INTRODUCTION}

Monitoring dairy cattle physiology and behavior has extensive implications within both research and onfarm settings. Dairy consumers and producers often express concern over animal well-being. Sleep is essential to the welfare of the animal and is vital to brain function and maintenance of homeostatic control (Hobson, 2005). According to Siegel (2005), sleep can be defined as a state of immobility with greatly reduced responsiveness. Both non-rapid-eye movement (NREM) and rapid eye movement (REM) sleep are homeostatically regulated in separate ways suggesting that each has important functions for optimal health. Increasing evidence suggests a clear link with the immune system and disease resistance. A hypnogram, discussed by Ruckebusch (1975), is a circadian sleep profile defined by brain waves from an electrocorticogram and indicates an animal's ecological niche, suggesting that sleep is an adaptation to its environment.

Sleep research in human medicine has advanced considerably in recent years by the development of activity-based monitoring systems (Chen et al., 2013). Although the basic physiological functions of sleep are not well understood, a breakthrough in this area has recently been made by Maiken Nedergaard's laboratory at Rochester University (Rochester, NY). A primary function of sleep appears to be flushing the brain of toxic waste products that accumulate during wake (Xie et al., 2013). This feature of sleep seems to be a fundamental and core function that may be critical to other sleep functions, including improved learning, memory, optimal immune function, endocrine function, and many aspects of general health.

Research on dairy cattle sleep is limited and has primarily consisted of studying brain electrophysiology 
through electroencephalography (EEG; Ruckebusch, 1965). Very few of these studies exist because of their cost and the physical invasiveness of the procedures. Dairy cattle sleep research has been limited because of the inability to accurately and easily measure sleep versus wake states. It could also be argued that most researchers have not thought to measure sleep. Current related research looks at lying time, which focuses on the quantity of resting behaviors rather than the quality of rest behaviors. Understanding and monitoring dairy cattle sleep under typical dairy farm conditions may improve health, increase milk production, and reduce involuntary culls resulting from disease (Gamaldo et al., 2012). Research from Ruckebusch (1972) used EEG and electrocorticographic patterns to classify slowwave sleep (characterized with low muscle tone) and paradoxical sleep (absence of muscle tone) along with drowsy states. Results showed that cows spend most of their time awake in a drowsy state when accustomed to their environment and are not sleeping or eating.

A noninvasive sleep monitoring system for mice has recently been developed for research purposes. This system relies on piezoelectric materials affixed to the floor of the mouse cage that function as exquisitely sensitive motion detectors. When the mice are asleep, virtually all movements are associated with respiration. When the mice are awake, even in "quiet wake," subtle postural adjustments and head movements disrupt the respiratory tract, and during active wake, large voluntary movements produce highly erratic wave patterns. (Donohue et al., 2008).

Understanding sleep in humans is still very limited and researchers have many questions with uncertain answers. Sleep deprivation in humans is followed by sleep compensation (Rechtschaffen, 1998) just as cows increase lying time after lying deprivation (Munksgaard and Simonsen, 1996). This pattern could possibly imply that cows may compensate for sleep after deprivation. More recently, new commercial technologies measuring sleep based on wrist movement (Fitbit, Fitbit Inc. San Francisco, CA; Jawbone UP, Jawbone, San Francisco, $\mathrm{CA}$ ) and stillness have become popular. Smartphone applications have also become a convenient way to track sleep behavior based on stillness (Chen et al., 2013). Montgomery-Downs et al. (2012) reported that the intradevice reliability for the Fitbit in comparison with standard actigraphy was 96.5 to $99.1 \%$. Both overestimated sleep efficiency and sleep time. The Fitbit appeared to be an acceptable activity measurement for the average population, but it should not be used to assess sleep disorders at this time because results can be misleading.
Similar to the current study, human sleep researchers are also seeking alternatives to invasive and expensive polysomnography sleep duration studies and instead are using wrist accelerometers. A study conducted by Jean-Louis et al. (2001) validated a wrist accelerometer (Actillume, Ambulatory Monitoring Inc., Ardsley, NY) against polysomnography and found an $85 \%$ agreement when tested on women (50-77 yr old) and a $91 \%$ agreement for young adults (19-34 yr old), proving the device to be effective.

The objective of the current study was to test if a noninvasive device can measure sleep-like behaviors in dairy cattle. The long-term objective is to continue developing this device to characterize sleep patterns in dairy cattle to assess sleep quality and how it relates to immune function, health, and productivity.

\section{MATERIALS AND METHODS}

The study was conducted at the University of Kentucky Coldstream Dairy using lactating Holstein ( $\mathrm{n}=$ 4) cows in September 2013. The cows were housed in a tiestall barn for the duration of the study. The barn consisted of tiestalls measuring $125 \mathrm{~cm}$ wide by $170 \mathrm{~cm}$ long with Dual Chamber Cow Waterbeds (Advanced Comfort Technology Inc., Reedsburg, WI) as the base. Kiln-dried sawdust $(5 \mathrm{~cm})$ was added for bedding twice daily. Cows were provided ad libitum water and TMR. Fans were on during most observation periods for ventilation. Natural light was used to observe cows during the day and at night, 2 barn lights created a dim light that allowed observers to see the cows, with the least disturbance.

\section{Live Observations}

Observers sat approximately $2 \mathrm{~m}$ directly behind the 4 cows. Human observer presence may have influenced the amount of sleep time. However, this influence would not affect the relationship between sleep behaviors recorded by human observers and the monitoring device.

Cows were observed continuously for two 24-h periods to record behavior. Two observers watched 2 cows during 4-h shifts. Observers were trained to recognize wake-, drowsy-, and sleep-like behaviors (Table 1).

\section{Accelerometer Monitor}

The 3-axis accelerometer device, developed by the coauthors, sampled at $32 \mathrm{~Hz}$. The system consisted of a low-cost battery-powered accelerometer that fit on a halter attached to the cow's neck (Figures 1 and 2). 


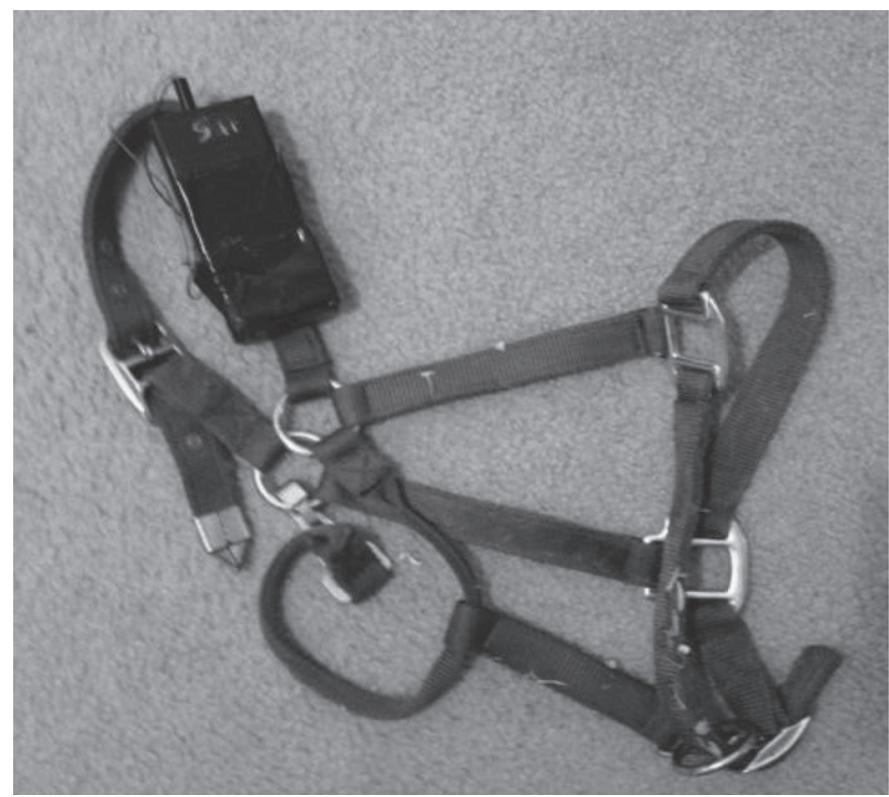

Figure 1. Accelerometer-based sleep device attached to harness.

Data were stored on a removable SD card. Two AA batteries located both internally and externally powered the device (Figure 1).

Observations were compared with movements shown in the accelerometer data. The study included 652 min of high-activity wake behaviors and $107 \mathrm{~min}$ of sleep-like behaviors as scored by human observers and synchronized with data from accelerometer device data from all 4 cows. This scoring was used to label sections of the signal for training and testing the classifiers. The training and testing phase used equal amounts for data in a bootstrapping analysis to estimate the mean performance and expected variation. Feature vectors were computed from a random selection of 20 segments of wake and 20 segments of sleep and were applied to create the classifier (training phase). Classifier performance was then tested with an independent set of 20 segments from each behavioral class for testing. This procedure

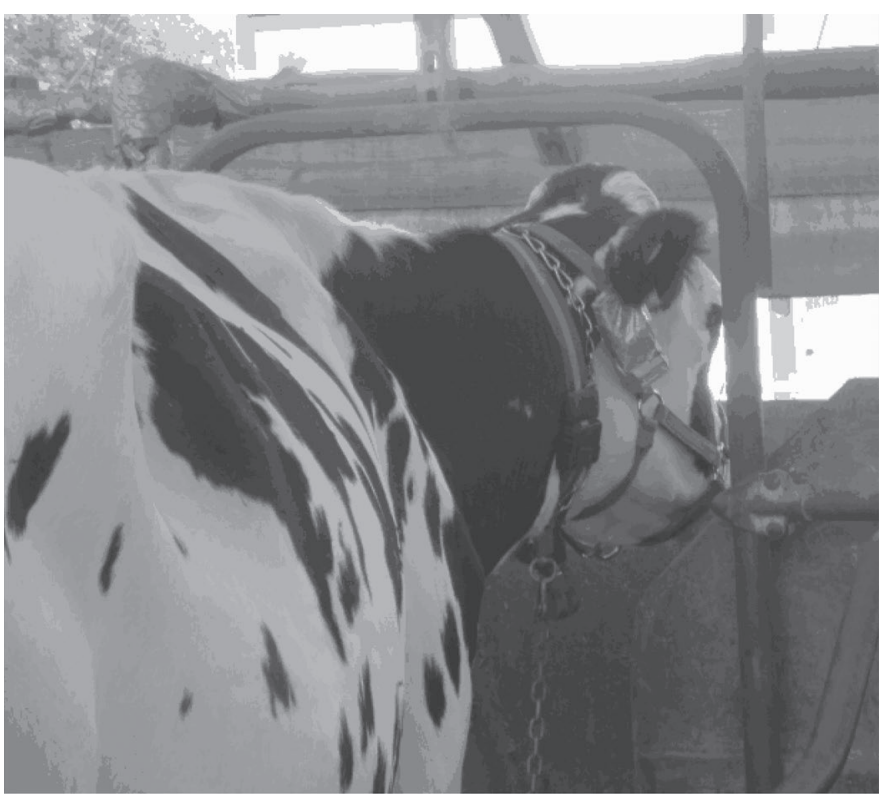

Figure 2. Accelerometer-based sleep device attached on the right side of the cow's neck.

was repeated 100 times, and the mean and standard deviation of classification rates were computed. Only sections for which the human observer was confident of a distinct behavior were used. This approach typically included longer epochs of the same behavior (at least $32 \mathrm{~s}$ or greater) to give time for observer to record the observation. Uncertain behavior transitions were not included to ensure consistency between the human observation clock and the accelerometer. This selection was completed before the classifier design and test phase.

The direct outputs of the 3 -axis accelerometer are voltages proportional to accelerations associated with motion in the $x, y$, and $z$ orthogonal directions. Because the orientation of the device on the side of the cow's neck was not identical for all cows, a motion signal independent of direction was derived. This signal cap-

Table 1. Description of visually observed behaviors used to validate an accelerometer-based sleep device indicating wake, drowsy, and sleep states of 4 cows during the observation period

\begin{tabular}{llll}
\hline Classification & Behavior & Head position & Open \\
\hline Wake & Standing or lying & Head up & \\
& Alert & & \\
Drowsy & Can be eating, drinking, or ruminating & Head up or relaxed & Partial or closed \\
Sleep-like & Standing or lying & & \\
& Still & Lying & Head on ground or flank \\
& Still & & Closed \\
\hline
\end{tabular}


tured the energy and force expended by the cow and quasiperiodic motions from rumination and breathing.

\section{Signal Features}

To explicitly describe the features tested in this study, specifically energies related to any motion independent of direction, equations are provided to show how features were computed from the 3 channels of the accelerometer data. The fundamental signal from which all features are derived is the calibrated omnidirectional motion signal, which is referred to as the radial signal. First, the device was calibrated so that voltage outputs corresponded to $g$ units. The radial signal, $R[n]$, was derived from $x, y$, and $z$ output voltages using the Euclidian sum:

$$
R[n]=\sqrt{x^{2}[n]+y^{2}[n]+z^{2}[n]}-1
$$

where $x[n], y[n]$, and $z[n]$ were discrete samples of the 3 outputs from the accelerometer. The 1 was subtracted to remove the $g$-force from gravity, so $R[n]=0$ corresponds to when the cow was motionless. This equation can now be used to track general motion, independent of direction.

Features tested in this work were computed from the energy in $R[n]$ and represented various level of activity and motion over various time intervals. The first feature is the root mean squared of $R[n]$ computed over short time intervals. Its computation was given by

$$
\sigma_{N}[n]=\sqrt{\frac{1}{N-1} \sum_{i=n}^{n+N-1}(R[i]-\mu[n])^{2}},
$$

where $N$ is the number of samples in the time interval, $n$ is the index at the start of the time interval, and $\mu[n]$ is given by

$$
\mu_{N}[n]=\frac{1}{N} \sum_{i=n}^{n+N-1} R[i]
$$

The actual feature was the standard deviation of the radial signal; however, the subtraction of 1 in Equation [1] results in a zero mean signal for most common motions over intervals of $20 \mathrm{~s}$ or greater. The feature in Equation [3] was typically low for stillness with shallow breathing and intermittent stirrings. The value was moderate for persistent motion such as rumination and heavy breathing, and the value was large for transient head motions from eating, locomotion, and looking around. Figure 3 shows examples of this feature for 3 general behavioral states. Notice the epochs of low activity with intermittent sparse spikes. These epochs were typical of the state showing sleep-like behaviors. The active states had 2 distinct patterns, drowsing and active. They are illustrated here because in the development of the classifier, both of these states need to be distinguished from sleep-like behaviors. This example suggests that drowsing (often accompanied by rumination and subtle motion) has a moderate signal level and sometime with periodic components, typical of rumina-

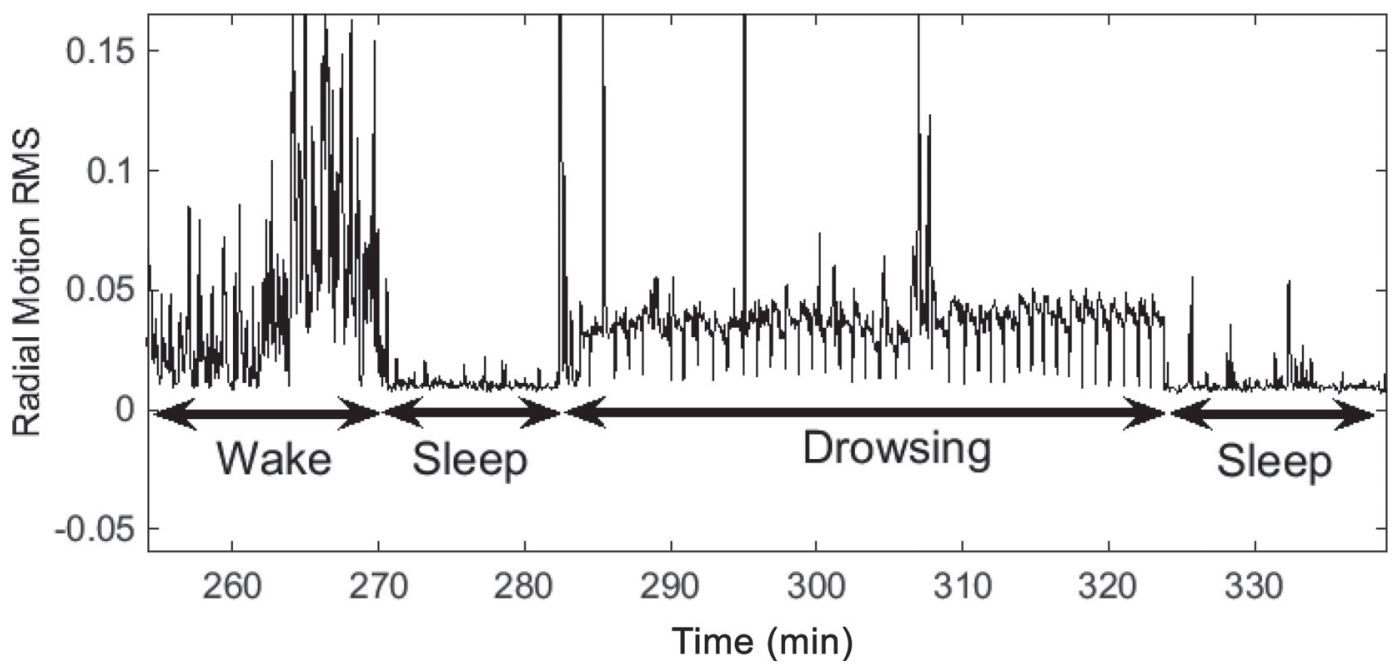

Figure 3. The radial signal (RMS = root mean square) from an accelerometer-based sleep device for $1 \mathrm{~h}$ from data used in this study illustrating typical patterns corresponding to human observed periods of wake, sleep, and drowsing. 
tion, and the other has large frequent peaks typical of locomotion, alert behavior, and eating.

Another related feature was the mean values of the $R[n]$ envelope peaks (local maxima). Peaks represent movement events; therefore, features based on peaks were an efficient way to characterize motion. Stronger peaks indicated movements with greater force; a greater number of peaks indicated more activity. This feature can be described mathematically as follows:

$$
p_{N}[n]=\frac{1}{K} \sum_{k=1}^{K} r_{k}
$$

where $p_{N}[n]$ is the average-radial-peak feature, $r_{k}$ is the amplitude of $k$ th local maximum of $R$ on interval $[n, n$ $+N-1$ ], and $k$ is the total number of local maxima in that interval.

\section{Linear Discriminant Analysis}

The radial signal features were examined for maximizing the performance of detecting sleep behavior using a Fisher's linear discriminant analysis (LDA) classifier (McLachlan, 2004; Donohue et al., 2008). Models were designed to correlate motion, as characterized through $\mathrm{R}$, with observed sleep and wake behaviors. The signal features were examined in an LDA classifier to discriminate sleep-like behaviors from all other wake behaviors.
The development of a classifier with the LDA requires a set of training data to determine a set of multipliers for each proposed feature, such that the sum of the multiplied features results in a value (decision statistic) that can separate the 2 classes with the minimal amount of error. To test if the classifier can work with data not trained on, an independent set of data must be used with the classifier to assess classification error.

The ability of the features to discriminate these behaviors was dependent on the window length $N$, used to compute the features. States of relative stillness occurred when the cow was alert and were similar to the stillness observed in sleep-like behavior. Figure 4 illustrates this, where $\sigma_{N}$ was computed over 4-s windows $(\mathrm{n}=128)$. Three types of behavior (from different times) were overlaid on the same plot for comparison. Note that the state of sleep-like behavior remains at a low level throughout the 350-s period. However, during the observed wake interval, up to 30-s intervals had stillness that was similar to sleep-like behavior. The drowsy behaviors were characterized by heavier breathing; subtle movements of rumination were at a consistent higher level with transient dips toward the level of the sleep-like behaviors. Although the initial thought that drowsing would be a greater challenge to distinguish from sleep, this result (shown at a higher time resolution than that in Figure 3) shows that for some periods of standing, the accelerometer picks up stillness similar to sleep-like behavior. However, although the

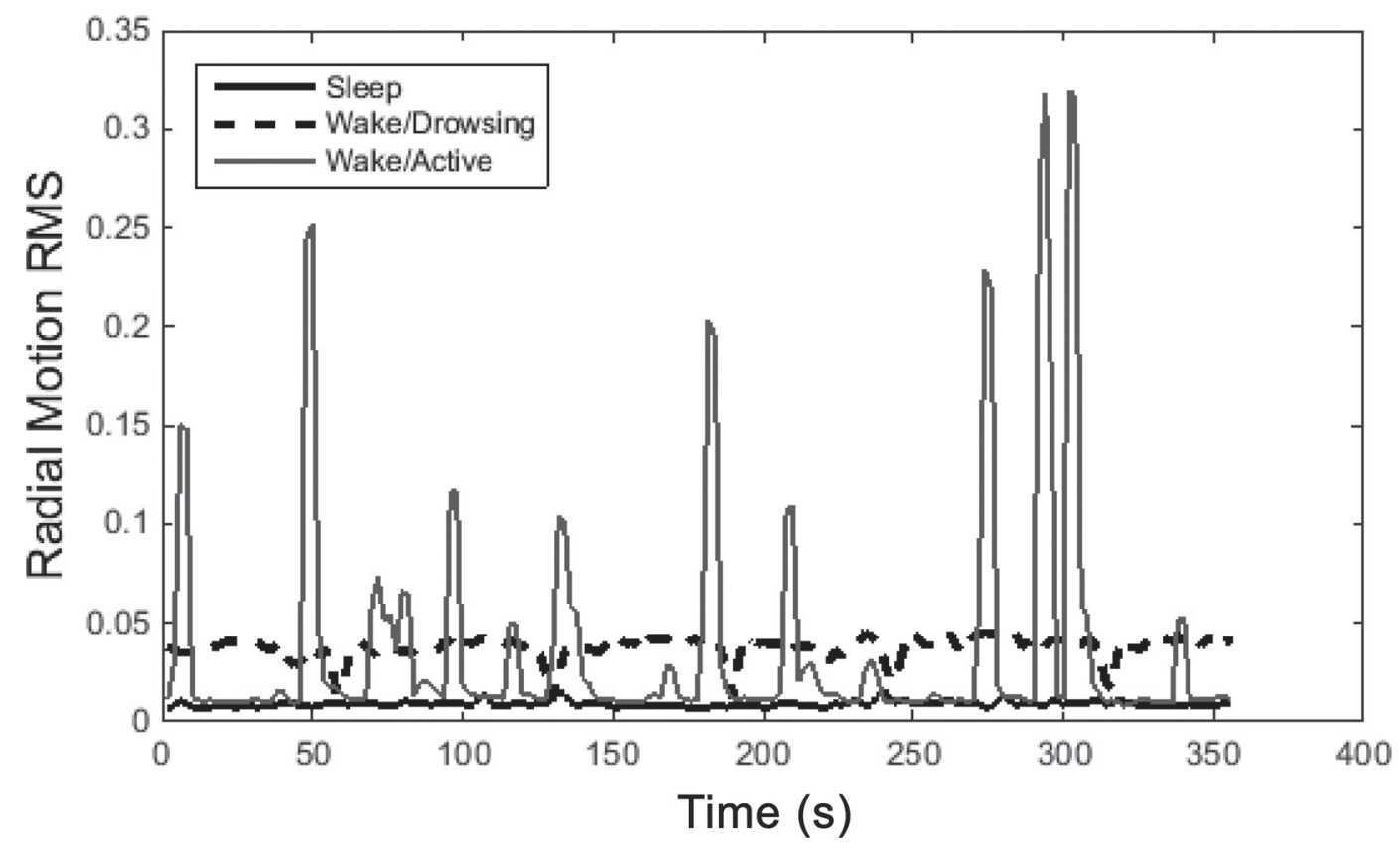

Figure 4. Radial acceleration standard deviation [root mean square (RMS) power] from an accelerometer-based sleep device computed over 4-s sliding windows. The peaks in these windows represent sudden movements such as a jerk of the head while the cow was alert. The wake/ drowsing line shows consistent dips indicating movements while ruminating, and the sleep line shows a state in which the cow was still. 


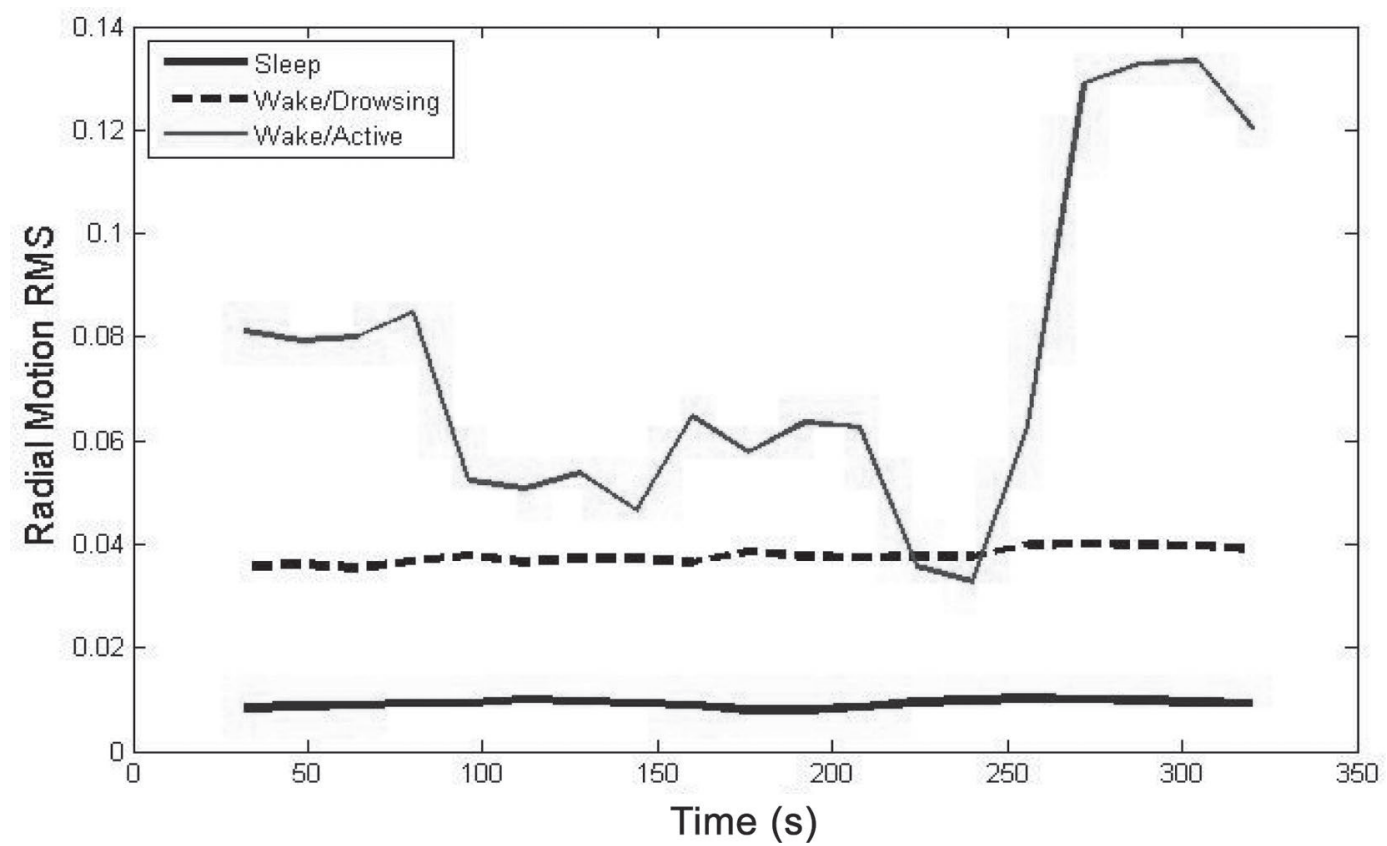

Figure 5. Radial acceleration root mean square (RMS) values from an accelerometer-based sleep device computed over 64-s sliding windows. The data from these windows show motivation for using a larger segment to compute RMS values. This larger segment allows recognition of the different levels of motion and separation between wake-active and sleep data.

movements recorded by the accelerometer in this wake state are less frequent than in other states, they are very strong. To enhance the ability of the feature to distinguish this state from a sleep-like state, the window size can be increased to increase the likelihood that a movement during the wake state will occur. Figure 5 compares the same periods with a 64-s interval used for computing $\sigma_{N}$. Note in this case a clear separation was apparent between wake and sleep-like behaviors. This finding suggests a trade-off between resolution and accuracy of the classifier. Therefore, the classifier was tested with multiple windows sizes $(32,64$, and 96 s) to better understand this trade-off.

For each window size tested, features were extracted and saved as feature vectors. In the case of the 2 features described in this paper, the feature vector $\mathbf{f}$ was

$$
\mathbf{f}=\left[\begin{array}{c}
\sigma_{N} \\
p_{N}
\end{array}\right] \text {. }
$$

where $\sigma_{N}$ is defined as in equation [2] and $p_{N}$ is defined as in equation [4]. The linear discriminate was computed from the training set vectors using

$$
\mathbf{v}=\boldsymbol{\Sigma}^{-1}\left(\mathbf{m}_{s}+\mathbf{m}_{w}\right)
$$

where $\mathbf{m}_{s}$ and $\mathbf{m}_{w}$ are the mean feature vectors computed over the sleep-like and wake segments, respectively, and $\boldsymbol{\Sigma}$ is the covariance matrix averaged between both classes. The classifier performance was tested with 20 independent feature vectors from each class (exclusive of those used in training) and applied to the following decision rule:

$$
\begin{aligned}
& \text { If } T_{d} \leq \mathbf{f}^{\mathrm{T}} \mathbf{v} \text {, decide sleep } \\
& \text { If } T_{d}>\mathbf{f}^{\mathrm{T}} \mathbf{v} \text {, decide wake, }
\end{aligned}
$$

where $T_{d}$ was the minimum error threshold and the superscript ${ }^{\mathrm{T}}$ denotes the transpose operation.

\section{Statistical Analysis}

A bootstrapping method was used to assess performance. Subsets of the feature vectors in each class were selected for training and testing multiple times to estimate the repeatability of the estimate. Sets of feature vectors for each testing and training phase are exclusive (i.e., vectors used to train classifier and not used in the testing to ensure the classifier generalizes and that was not simply learning the data set). In this analysis the training and testing phase was repeated 100 times. The mean correct classification rate for each class and the 95\% confidence limits were computed for 3 different window sizes. Features were applied individually and in combination with each other. 
Table 2. The mean correct classification rate for each class and the computed $95 \%$ confidence limits for features extracted from different window lengths $( \pm 95 \%$ CI $)$ from an accelerometer-based sleep device

\begin{tabular}{lcccc}
\hline Feature $^{1}$ & $\begin{array}{c}\text { Window } \\
\text { length (s) }\end{array}$ & $\begin{array}{c}\text { Agreement: sleep-like } \\
\text { behaviors (\%) }\end{array}$ & $\begin{array}{c}\text { Agreement: wake } \\
\text { behaviors (\%) }\end{array}$ & $\begin{array}{c}\text { Overall } \\
\text { agreement (\%) }\end{array}$ \\
\hline$p_{N}$ & 32 & $91.1 \pm 1.1$ & $86.4 \pm 1.2$ & $88.8 \pm 1.2$ \\
$\sigma_{N}$ & 32 & $90.5 \pm 1.2$ & $87.4 \pm 1.3$ & $88.9 \pm 1.3$ \\
{$\left[\sigma_{N}, p_{N}\right]$} & 32 & $90.6 \pm 1.2$ & $85.6 \pm 1.2$ & $89.0 \pm 1.3$ \\
$p_{N}$ & 64 & $92.2 \pm 1.0$ & $92.5 \pm 1.0$ & $92.4 \pm 1.0$ \\
$\sigma_{N}$ & 64 & $92.3 \pm 1.1$ & $93.8 \pm 0.9$ & $93.1 \pm 1.0$ \\
{$\left[\sigma_{N}, p_{N}\right]$} & 64 & $91.5 \pm 1.0$ & $91.3 \pm 1.0$ & $92.7 \pm 1.0$ \\
$p_{N}$ & 96 & $97.0 \pm 0.6$ & $95.5 \pm 0.9$ & $96.2 \pm 0.8$ \\
$\sigma_{N}$ & 96 & $96.9 \pm 0.7$ & $95.5 \pm 0.8$ & $96.2 \pm 0.7$ \\
{$\left[\sigma_{N}, p_{N}\right]$} & 96 & $96.4 \pm 0.7$ & $94.8 \pm 0.9$ & $95.6 \pm 0.8$ \\
\hline
\end{tabular}

${ }^{1}$ Where $p_{N}$ is the average of the local maxima of radial activity signal envelope over local intervals of $N$ samples given in equation [4]; $\sigma_{N}$ is the means square of the radial activity signal over local intervals of $N$ samples given in equation [2]; $\left[\sigma_{N}, p_{N}\right]$ is the feature vector comprising these 2 individual features, as given in equation [5].

\section{RESULTS AND DISCUSSION}

Results from the bootstrapping analysis between human observation and the LDA are presented in Table 2. As expected, the best overall performance came from the large window size with $96.2 \pm 0.7 \%$ agreement when using $\sigma_{N}$ by itself; however, $p_{N}$ and the combined set (feature vector) were within each other's $95 \%$ confidence limits. The smallest window, $32 \mathrm{~s}$, achieved a classification agreement of $89.0 \pm 1.3 \%$ for the combined features. All cases showed no statistical difference between using the feature individually or combined in a vector. All were within their 95\% confidence ranges. In addition, no strong imbalances between classification errors in the sleep-like or wake state were found. The greatest difference between sleep-like behaviors and wake accuracy came from the 32 -s window showing a 3 to $5 \%$ difference favoring more accuracy for the sleeplike state.

Although the use of a larger window size improved accuracy, it lowered the resolution of making a sleepwake decision. The shorter 4-s window had a higher resolution and showed specific peaks in each line, where the larger window was accurate in showing the difference in the radial signal between the sleep-like behaviors and the wake behaviors (Figure 4). Sleeplike bouts of around $20 \mathrm{~s}$ would likely not be detected with the 64-s window (Figure 5) but would be detected with the 32-s window, because the latter could pick up shorter bouts of sleep-like behaviors. To improve resolution, additional features that contribute independent information are needed. Other features from the radial signal were initially examined, such as entropy and spectral peak statistics, but these features showed little to no correlation with the observed sleep-like and wake states. Most of the performance was driven by movements detected in the neck area, which is similar to past work (Hänninen et al., 2008). Both features used were highly correlated, which explains why their combination in a feature vector did not result in a significant improvement. Other features that are independent or complementary would be needed for exploring improvements in accuracy. The current device was limited to detecting motion independent of direction and orientation. More effective mounting procedures, multiple accelerometers, and other biological estimates from the animal could potentially improve performance and allow multiple behaviors to be detected and tracked, but this would potentially increase the complexity of the device and mounting procedures.

The results and analyses in this work are limited by the use of human observation to label sleep-like behaviors and wake behaviors. Short sleep-like bouts would likely be missed by human observation. If the observations recorded by the observer did not show consistency with sleep-like or wake behaviors, those data were not used in the classifier. In addition, identifying when the cow actually went into a sleep-like state after going into a sleep-like posture was not clear. More elaborate EEG studies would be needed to address these issues.

Sleep in animals is often estimated by behavioral observations or recorded on restrained animals with invasive EEG techniques that might affect sleep patterns (Ternman et al., 2012). In the current study, the accelerometer was placed on a halter, providing minimal irritation to the animals, thus minimally affecting sleep patterns. The change in surroundings from a freestall barn, where these cows were normally housed, to a tiestall barn could have altered sleeping times. Human presence also could have reduced normal amounts of sleep. Previous studies using observed behaviors validated with EEG recordings provided subjective classification methods of behaviors shown in Table 1 as sleep versus wake behaviors in calves but not cows (Hän- 
ninen, 2007; Ternman et al., 2012). These researchers demonstrated that the behaviors were not as useful for cows as for calves.

In a similar study, an accelerometer was used to classify calf sleep and wake behavior while also looking at NREM and REM sleep stages (Hokkanen et al., 2011). The model was able to distinguish $66 \pm 8 \%$ and $70 \pm 6 \%$ of the total time the calves were in NREM and REM sleep, respectively, but overestimated the duration of NREM sleep and underestimated REM duration. Video tapings were used instead of human observation, which may have made it difficult to see eyes opening and closing. The results were similar to the current study, with drowsy behavior being difficult to classify in the analysis. The data were combined and analyzed as wake-drowsy and wake-active behaviors. Lying behavior technologies would also have this issue because they do not provide indications of a drowsy state. Although a drowsy state alone was not indicated often by the accelerometer data, signals from the monitors picked up rumination (Figure 4). According to Ruckebusch (1972), slow waves from an encephalogram recording corresponded with some rumination behavior, suggesting the possibility of partial sleep during rumination.

In relation to, but contrasting to sleep behavior research, many studies have focused on lying time (Munksgaard and Simonsen, 1996; Fisher et al., 2002; Cooper et al., 2008). Multiple sensors (e.g., IceTag, IceRobotics Ltd., Roslin, Scotland, UK; TinyTag, Gemini Dataloggers Ltd., Chichester, UK; AfiAct Pedometer Plus, Afimilk, Kibbutz Afikim, Israel) have been successfully used to measure cow lying time. If a cow is deprived of lying time, her welfare may be reduced (Haley et al., 2000; Fregonesi and Leaver, 2001; Cooper et al., 2008). Cows deprived of lying time have shown physiological and behavioral signs of stress (Munksgaard and Simonsen, 1996; Fisher et al., 2002; Cooper et al., 2008). Cows prioritize lying down, or resting, even over the opportunity to eat (Munksgaard et al., 2005). Although the amount of time a cow spends lying can provide insight on how housing and management affect cow comfort and physiological status, lying time focuses only on rest quantity without assessing rest quality, which was the focus of the current study.

Limitations of the experiment included battery life and recording consistency of the monitors. Development of future versions of the device will include consideration of alternative battery locations and a protective cover. Data analysis was limited because of complications with the monitors and because they could not get wet; however, enough data were provided to validate sleep-like behavior and sleep time.
Successfully classifying sleep-like behavior with this device demonstrates proof-of-concept testing. Future devices may also include measures of respiration (Donohue et al., 2008) and heart rate, which is said to decrease in the transition from alert wakefulness to paradoxical sleep, similar to REM sleep (Ruckebusch, 1972).

Future validation efforts should include comparisons with EEG measurements of sleep. Final versions of this device could be used to compare sleep quantity and quality and the amount of sleep among different management systems and facility designs and to assess immunological effects.

This initial proof-of-concept study was a first attempt to determine if head movements assessed by an accelerometer may be a useful measure for cow sleep and wake states, and in the future, cow respiratory variables (which aside from useful physiological information, may be used to further improve cow sleep and rest assessments). Future studies will be conducted with EEG or electromyography (EMG) validation. However, cow sleep, even with the gold standard of EEG or EMG measurements, has been difficult to determine because of multiple factors. Ruckebusch (1972) using EEG or EMG arbitrarily defined a "drowsy" state as wake, when it may contain considerable sleep, perhaps a light form of sleep analogous to human stage 1 or even lighter. Likewise, considerable light sleep may occur during rumination, which can disrupt EEG or EMG measurements. Eye closure does not necessarily correlate with sleep (Ruckebusch, 1972). Future studies will need to use a variety of measures to assess sleep, including EEG or EMG, but also include arousal threshold, which is arguably a more fundamental feature of sleep than even EEG or EMG and applies to all animals. We included features of posture and eye closure in assessing our sleep-like behaviors because it was likely that eye closure in addition to postural changes represent sleep or a sleep-like state more consistently, even if some sleep occurs with eyes partially open or in a variety of postures (even standing).

Defining management and housing factors that affect sleep may be important for improving animal health and well-being. In turn, this information could be used to design dairy facilities supportive of increased sleep. These concepts have been applied to other mammals to a limited extent, but research in adult cows is limited.

\section{CONCLUSIONS}

This newly developed proof-of-concept device was successfully tested for classifying sleep-like behaviors compared with human observation. However, it needs 
to be validated with an EEG to determine if the cow is in a true sleep state. Overall, it could provide valuable information for knowing that the cow is getting "quality rest" based on those behaviors even if we do not know for sure that she is actually in a true sleep state. The accuracy of the device in this study suggests that this type of device could be used to track animal health and well-being in research and on-farm in the future, but at this point it could not be used in large settings.

\section{REFERENCES}

Chen, Z., M. Lin, F. Chen, N. D. Lane, G. Cardone, R. Wang, T. Li, Y. Chen, T. Choudhury, and A. T. Campbell. 2013. Unobtrusive sleep monitoring using smartphones. Page 145-152 in 2013 7th International Conference on Pervasive Computing Technologies for Healthcare and Workshops, Venice. Institute of Electrical and Electronics Engineers, New York, NY.

Cooper, M. D., D. R. Arney, and C. J. C. Phillips. 2008. The effect of temporary deprivation of lying and feeding on the behaviour and production of lactating dairy cows. Animal 2:275-283.

Donohue, K. D., D. C. Medonza, E. R. Crane, and B. F. O'Hara. 2008. Assessment of a non-invasive high-throughput classifier for behaviours associated with sleep and wake in mice. Biomed. Eng. Online 7:14.

Fisher, A. D., G. A. Verkerk, C. J. Morrow, and L. R. Matthews. 2002. The effects of feed restriction and lying deprivation on pituitary-adrenal axis regulation in lactating cows. Livest. Prod. Sci. 73:255-263.

Fregonesi, J. A., and J. D. Leaver. 2001. Behaviour, performance and health indicators of welfare for dairy cows housed in strawyard or cubicle systems. Livest. Prod. Sci. 68:205-216.

Gamaldo, C. E., A. K. Shaikh, and J. C. McArthur. 2012. The sleepimmunity relationship. Neurol. Clin. 30:1313-1343.

Haley, D. B., J. Rushen, and A. M. de Passille. 2000. Behavioural indicators of cow comfort: Activity and resting behaviour of dairy cows in two types of housing. Can. J. Anim. Sci. 80:257-263.

Hänninen, L. 2007. Sleep and rest in calves - Relationship to welfare, housing and hormonal activity. PhD Diss. Department of Production Animal Medicine, University of Helsinki, Finland.
Hänninen, L., J. P. Mäkelä, J. Rushen, A. M. de Passillé, and H. Saloniemi. 2008. Assessing sleep state in calves through electrophysiological and behavioural recordings: A preliminary study. Appl. Anim. Behav. Sci. 111:235-250.

Hobson, J. A. 2005. Sleep was of the brain, by the brain and for the brain. Nature 437:1254-1256.

Hokkanen, A.-H., L. Hänninen, J. Tiusanen, and M. Pastell. 2011. Predicting sleep and lying time of calves with a support vector machine classifier using accelerometer data. Appl. Anim. Behav. Sci. 134:10-15.

Jean-Louis, G., D. F. Kripke, R. J. Cole, J. D. Assmus, and R. D. Langer. 2001. Sleep detection with an accelerometer actigraph: Comparisons with polysomnography. Physiol. Behav. 72:21-28.

McLachlan, G. J. 2004. Discriminant Analysis and Statistical Pattern Recognition. Wiley Interscience, Hoboken, NJ.

Montgomery-Downs, H. E., S. P. Insana, and J. A. Bond. 2012. Movement toward a novel activity monitoring device. Sleep Breath. 16:913-917.

Munksgaard, L., M. B. Jensen, L. J. Pedersen, S. W. Hansen, and L. Matthews. 2005. Quantifying behavioural priorities-Effects of time constraints on behaviour of dairy cows, Bos taurus. Appl. Anim. Behav. Sci. 92:3-14.

Munksgaard, L., and H. B. Simonsen. 1996. Behavioral and pituitary adrenal-axis responses of dairy cows to social isolation and deprivation of lying down. J. Anim. Sci. 74:769-778.

Rechtschaffen, A. 1998. Current perspectives on the function of sleep. Perspect. Biol. Med. 41:359-390.

Ruckebusch, Y. 1965. Electroencephalography in animals [abridged]. Proc. R. Soc. Med. 58:551-552.

Ruckebusch, Y. 1972. The relevance of drowsiness in the circadian cycle of farm animals. Anim. Behav. 20:637-643.

Ruckebusch, Y. 1975. The hypnogram as an index of adaptation of farm animals to changes in their environment. Appl. Anim. Ethol. $2: 3-18$.

Siegel, J. M. 2005. Clues to the functions of mammalian sleep. Nature 437:1264-1271.

Ternman, E., L. Hänninen, M. Pastell, S. Agenäs, and P. P. Nielsen. 2012. Sleep in dairy cows recorded with a non-invasive EEG technique. Appl. Anim. Behav. Sci. 140:25-32.

Xie, L., H. Kang, Q. Xu, M. J. Chen, Y. Liao, M. Thiyagarajan, J. O'Donnell, D. J. Christensen, C. Nicholson, and J. J. Iliff. 2013. Sleep drives metabolite clearance from the adult brain. Science 342:373-377. 